\title{
A qualitative investigation into the acceptability of a food-based rewards system in secondary schools
}

\author{
C. Rooney ${ }^{1}$, C.E. Neville ${ }^{1}$, J. Hanvey ${ }^{2}$, F. Kee ${ }^{1}$, C.T McEvoy ${ }^{1}$, S. Moore ${ }^{1}$, J.V. Woodside ${ }^{1}$ and \\ M.C. McKinley ${ }^{1}$ \\ ${ }^{1}$ Centre for Public Health, Queen's University Belfast, Belfast BT12 6BJ, UK and \\ ${ }^{2}$ Public Health Agency and Belfast Education and Library Board, Belfast, UK
}

The diet of UK children is currently suboptimal ${ }^{(1)}$. Hence, there is a need to develop effective means of encouraging young people to choose a healthier diet. Using rewards may be an effective method to positively influence young people's eating behavior ${ }^{(2,3)}$, but evidence regarding this approach is currently limited. In response to this, a scheme called 'eat4treats (E4T)' was developed to test the feasibility of a food-based reward system in secondary schools. The aim of the present study was to qualitatively assess the acceptability of the E4T scheme among the pupils who took part.

E4T was a non-randomised, controlled, parallel-group feasibility study. Three secondary schools (two intervention and one control) serving areas of the highest social deprivation in Northern Ireland were recruited, and year 9 and 10 pupils (boys and girls aged 12-14 years) within the schools were invited to take part. The two intervention schools implemented the E4T scheme in the school canteen, the control school canteen operated as normal. Pupils registered for E4T using their school email and password. During the four month trial, pupils taking part earned points for foods purchased at the school canteen, with better nutritional choices having a higher value. Pupils could exchange the points they earned for rewards (e.g. stationery, iTunes vouchers, sports equipment) via the E4T website. Semi-structured focus groups $(n=5)$ were used to evaluate the acceptability of the scheme among pupils $(n=35)$ in the two intervention schools at the end of the study. Discussions were recorded digitally, transcribed verbatim and analysed thematically by two independent researchers. Coded data was managed using NVivo 10 (QSR Intern, Melbourne, Australia).

Findings from the focus groups suggested that the overall concept of E4T was well received by pupils and there was a high degree of satisfaction with the rewards available, the study name, the logo, the study website and the promotional materials. However, pupils made a number of suggestions about how to improve the scheme including reviewing the registration process and enhancing engagement with the scheme after its launch. The table below outlines the main suggestions made by pupils.

Suggestions from pupils to improve the E4T scheme

- Make the registration process more straightforward as some pupils had difficulty activating their E4T account due to unfamiliarity with their school email and password. - Provide written reminders of website usernames and passwords to make sign-in easier.

- Frequent reminders about the scheme (e.g. automated messages via a phone App or text messages to pupil's phones if parents were happy to allow this).

- Implement a more efficient method for reward retrieval - rewards were ordered by the research team and delivered to schools which introduced some delays in pupils receiving them.

- Offer group rewards (e.g. for classes) in addition to individual rewards to introduce a competitive element.

This feasibility study demonstrated a high degree of acceptability for the E4T scheme among pupils and highlighted a number of important recommendations for improving the scheme that should be taken into consideration in the design of a pilot trial.

1. Bates B, Cox L \& Nicholson S (2016) NDNS results from years 5 and 6 combined of the rolling programme for 2012 and 2013 to 2013 and 2014. London: PHE \& FSA

2. Hendy H, Williams K \& Camise T (2005) Appetite 45, 250-263.

3. Horne PJ, Tapper K, Lowe CF, et al. (2004) Eur J Clin Nutr 58, 1649-1660. 